\title{
Bandwidth Enhancement of Microstrip Patch Antenna Using Complementary Rhombus Resonator
}

\author{
Lei Tao, Jianchun Xu, Haihong Li, Yanan Hao, Shanguo Huang $\mathbb{D}$, Ming Lei, and Ke Bi $\mathbb{D}$
}

State Key Laboratory of Information Photonics and Optical Communications, School of Science, Beijing University of Posts and Telecommunications, Beijing 100876, China

Correspondence should be addressed to Shanguo Huang; shghuang@bupt.edu.cn and Ke Bi; bike@bupt.edu.cn

Received 31 March 2018; Accepted 30 July 2018; Published 12 August 2018

Academic Editor: Eva Antonino-Daviu

Copyright (C) 2018 Lei Tao et al. This is an open access article distributed under the Creative Commons Attribution License, which permits unrestricted use, distribution, and reproduction in any medium, provided the original work is properly cited.

By using complementary rhombus resonator (CRR), a small-size and low-profile microstrip patch antenna (MPA) with broad bandwidth has been proposed. Parametric studies were conducted to illustrate the working principle of the proposed antenna. An additional resonance is introduced by the CRR to broaden the bandwidth. Compared with the MPA without the CRR, the bandwidth of the proposed antenna is increased by $200 \%$. The measured results are in good agreements with the simulated ones, which demonstrate that this design provides a way to obtain the broadband antenna.

\section{Introduction}

Nowadays, with the rapid development of microwave technology, there is a growing demand on the bandwidth [1-5]. Microstrip patch antenna (MPA) is more capable than other antennas due to its small size, easiness of integration, light weight, and low profile, which can be used in the field of radar technology, space science, biomedical research, and wireless communication systems [6, 7]. However, compared with conventional microwave antennas, MPAs also have some limitations such as low gain, large ohmic loss in the array, and radiation of energy mostly to half space. Among them, the narrow bandwidth is the main drawback that hinders MPAs from practical application.

During the past years, numerous methods have been proposed to enhance the bandwidth of MPAs, such as increasing the thickness of the substrate $[8,9]$, decreasing the substrate dielectric constant [10,11], loading chip resistor [12], using parasitic patches in single layer and multilayers configuration [13-15], employing electromagnetic band gap structures [16$19]$, and using backed edge-fed cavity [20, 21]. However, most of these MPAs are made by adding additional structure so that their structures become more complex. Recently, complementary structures have been applied successfully in the design of various microwave devices [22-26]. Yoo et al. [27] studied a waveguide-fed metasurface antennas loaded with complementary metamaterial element and realized a low-loss and high radiation power properties. Cheng et al. [28] fabricated a self-packaged folded patch antenna loaded with a complementary split-ring resonator and obtained a $74 \%$ size reduction. The complementary structure provides a way to design the integrated devices with desired properties.

In this work, a MPA loaded with CRR is designed and fabricated for bandwidth enhancement. The CRR unit cell can resonate with the electromagnetic wave and significantly improve bandwidth performance of the MPA. The measured results are in concordance with the simulated ones, which manifest that the proposed antenna can be a good candidate for practical wideband applications.

\section{Structure and Principle}

Figure 1 shows the schematic diagram of the CRR-based MPA. It is fabricated on an $a \times b\left(40 \times 49 \mathrm{~mm}^{2}\right) \mathrm{FR} 4$ substrate with a thickness of $1.0 \mathrm{~mm}$ and a relative permittivity of 2.65 . The thickness of the copper layers is $0.035 \mathrm{~mm}$. The top layer of the proposed antenna consists of a rectangular patch with a small size of $w \times l$ and two different-width feedlines. The widths of the feedlines are $w f_{1}$ and $w f_{2}$, which are designed to achieve a $50 \Omega$ impedance matching. The CRR with the side length of $s$ and included angle of $\theta$ is etched in the ground plane. The slit width of the CRR is $1 \mathrm{~mm}$. The $d$ is the distance 


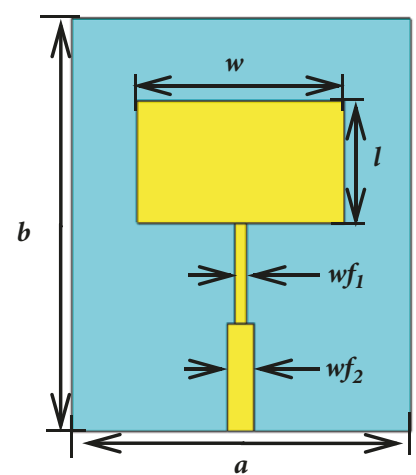

(a)

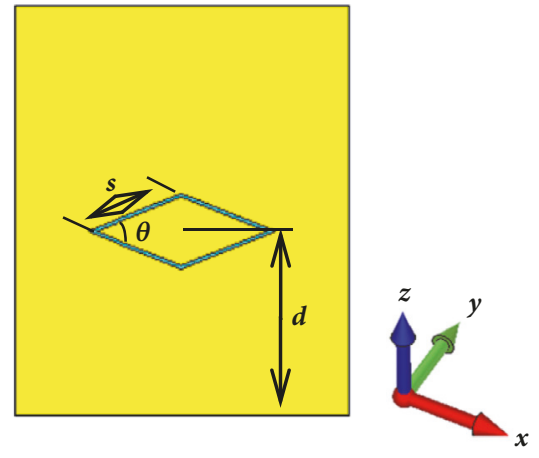

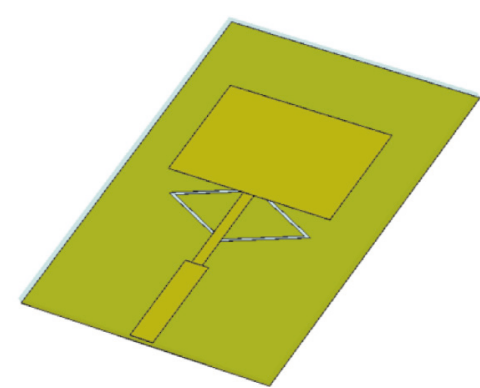

(b)

FIGURE 1: Schematic diagram of the CRR-based antenna. (a) Top and bottom views. (b) Three-dimensional view.

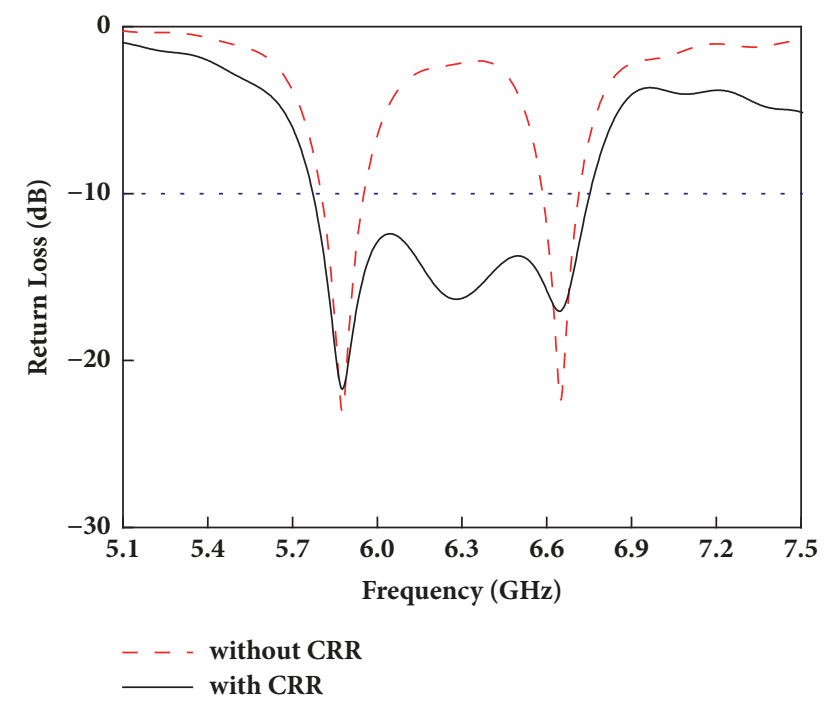

FIGURE 2: Simulated return loss of the proposed antenna with and without the CRR.

between the center of the CRR and the edge of the substrate. It is clearly seen that the CRR is deposited underneath the feedline, which is employed as a portion of combination with a microstrip line to make new resonance to achieve high magnetic coupling.

In order to observe the influence of the CRR unit cell on antenna performance, the return losses of the MPA without and with the CRR were investigated using the software CST Microwave Studio, as shown in Figure 2. It can be seen that, for the MPA without the CRR, only two resonant modes are excited at $5.8 \mathrm{GHz}$ and $6.65 \mathrm{GHz}$, respectively, with good impedance matching. For the MPA with the CRR, however, additional resonant mode at about $6.3 \mathrm{GHz}$ is excited due to the mutual coupling of the MPA and the CRR. These three frequencies are close to each other and combined to form a broadband, thus increasing the bandwidth of the antenna. The simulated impedance bandwidths below $-10 \mathrm{~dB}$ without the CRR are $0.2 \mathrm{GHz}(5.75-5.95 \mathrm{GHz})$ and $0.13 \mathrm{GHz}$ $(6.58-6.71 \mathrm{GHz})$. While adding the CRR, the bandwidth turns to $0.99 \mathrm{GHz}(5.77-6.76 \mathrm{GHz})$. It can be inferred that the combination of the MPA and the CRR can lead to bandwidth enhancement.

To understand the underlying physics of the CRR-based MPA, the electromagnetic properties of the CRR unit cell were studied. Figure 3(a) shows the transmission and reflection spectra of the CRR. The inset shows the schematic diagram of the CRR unit cell structure. It is composed of one FR4 substrate and one copper layer. The CRR is etched in the center of the copper layer. The propagation of the incident electromagnetic wave is set along the $y$ axis, and the electric field and magnetic field are set along the $z$ and $x$ axes, respectively. It can be seen that a resonance is obtained at $5.02 \mathrm{GHz}$. In order to further demonstrate the resonance phenomenon, the phase spectra of the CRR is shown in Figure 3(b). At $5.02 \mathrm{GHz}$, the transmission phase falls to its minimum and the maximum variation of phase is larger than $300^{\circ}$, illustrating that there exists a strong phase shifting effect, which further proves that a frequency response appears at $5.02 \mathrm{GHz}$.

In order to further analyse the working principle of CRR, we observed the electric field and current distribution of CRR at $5.02 \mathrm{GHz}$ as shown in Figure 4. From Figure 4(a), it is observed that the electric field is mainly concentrated around the upper and lower vertex regions of the CRR. Meanwhile, the electric field distribution at the left and right vertex regions of the CRR is very sparse. It indicates that the electric field mainly spreads along the upper and lower directions of the CRR, which is responsible for radiating power into free space. Figure 4(b) shows the current distribution of the CRR. The currents are centralized at the left and right vertex regions, which are induced by the magnetic-fields. In addition, we can observe that the included angle of the CRR has an impact on the distribution of currents. Based on the above analysis, we find that the unit cell structure is conducive to radiate energy at resonant frequency. Therefore, the CRR can be used to enhance the bandwidth of the antenna.

\section{Results and Discussion}

Figure 5 shows the simulated return loss of the proposed antenna with a series of $d, \theta, l$, and $w$. In the process of observing the influence of parameters, only one parameter 


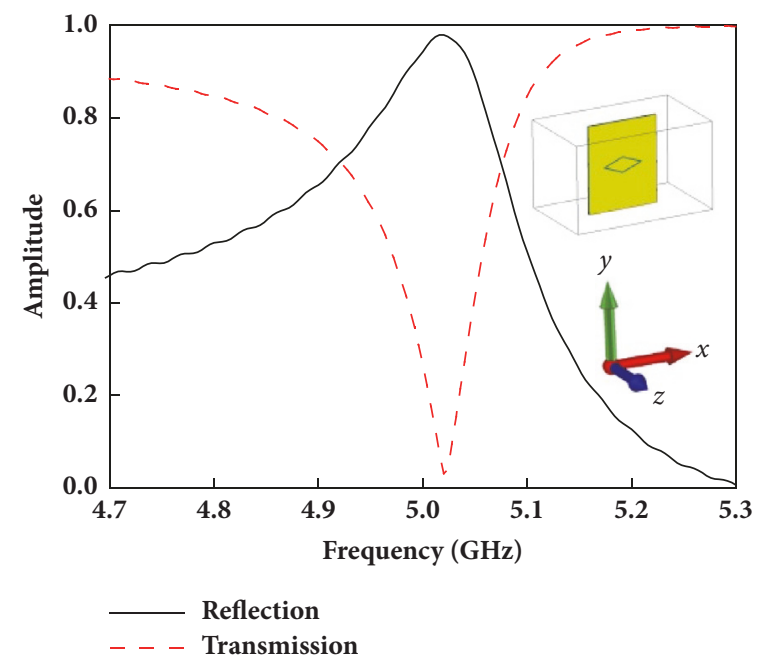

(a)

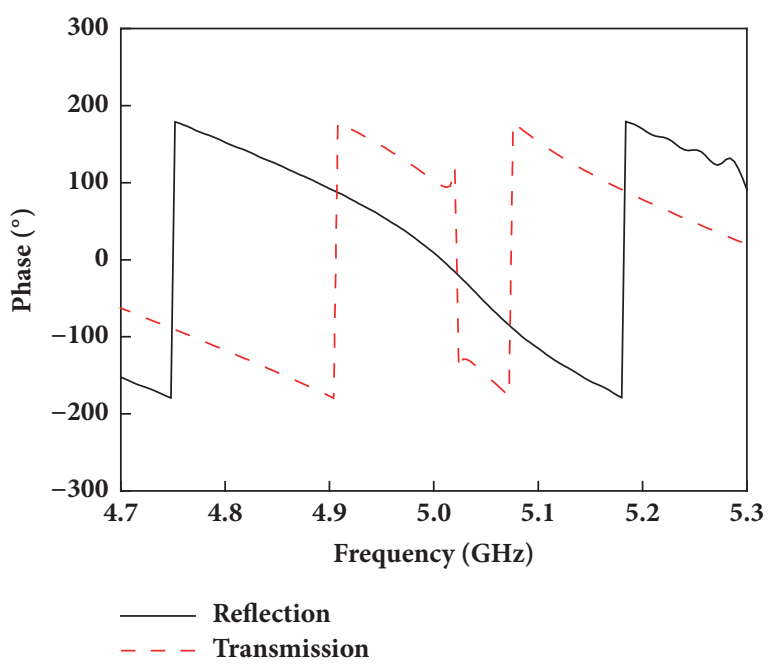

(b)

FIgURE 3: (a) Transmission and reflection spectra of the CRR. (b) Phase spectra of the CRR. The inset shows the schematic diagram of the CRR.

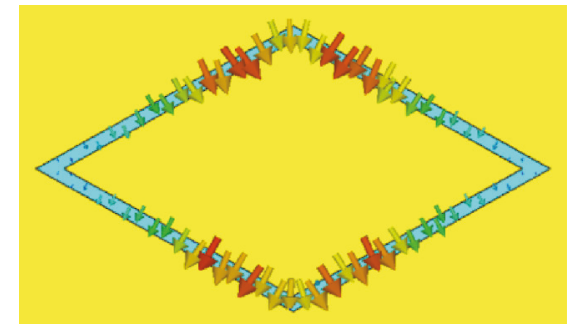

(a)

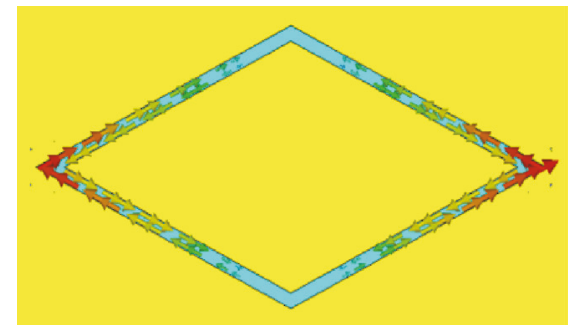

(b)

FIGURE 4: (a) Electric field distribution and (b) current distribution of the CRR.

is changed, and the other parameters are kept the same. As shown in Figure 5(a), it is observed that as $d$ increases from $21.4 \mathrm{~mm}$ to $22.0 \mathrm{~mm}$, the first frequency and the third frequency increase slightly. The second resonant frequency shifts to the lower part, which illustrates that the second frequency is related to the position of CRR. Since the currents on the ground are mainly concentrated below the feedlines and the radiating patch, different positions of the CRR may affect the current distribution on the ground, thus affecting the coupling between the radiating patch and the CRR, which in turn affect the resonant frequency. Figure 5(b) shows that as the included angle $\theta$ of the CRR decreases from $48.4^{\circ}$ to $43.6^{\circ}$ with an increment of $1.6^{\circ}$, the second resonant frequency becomes smaller. However, there are no apparent influences on the first frequency and the third frequency. It is inferred that the variation of the included angle can affect the current distribution on the ground, thus affecting the second frequency. Figure 5(c) demonstrates that when the length $l$ of the radiating patch increases from $14.5 \mathrm{~mm}$ to $14.8 \mathrm{~mm}$, the first resonant frequency moves to the lower frequency. In the meantime, the second frequency and the third frequency remain almost unchanged. As displayed in Figure 5(d), it can be observed that when the width $w$ of the radiating patch increases from $25.0 \mathrm{~mm}$ to $26.5 \mathrm{~mm}$, the third resonant frequency becomes smaller, and the first frequency and the second frequency do not change. After analysing the above parameters of the proposed antenna, the antenna design process is summarized: the first resonant frequency is controlled by the length $l$ of the radiating patch, and the second resonant frequency is controlled by the distance $d$ and the included angle $\theta$ of the CRR. The third frequency is controlled by the width $w$ of the radiating patch. The final optimized parameters are as follows: $w=25 \mathrm{~mm}, l=$ $14.5 \mathrm{~mm}, w f_{1}=1.6 \mathrm{~mm}, w f_{2}=3.2 \mathrm{~mm}, s=12 \mathrm{~mm}, \theta=43^{\circ}$, and $d=24.5 \mathrm{~mm}$. In the meantime, the maximum impedance bandwidth below $-10 \mathrm{~dB}$ of the proposed antenna is $1.1 \mathrm{GHz}$.

In order to figure out if there exists any numerical function that can describe the relationship between the antenna parameters and the return loss, the following research was carried out. First of all, the resonance modes of the antenna without CRR were studied. According to cavity theory, the radiation patch and the ground can be considered as a resonant cavity. The upper and bottom layers are PEC with a small size of $l \times w$, and the back and forth and left and right parts of the resonant cavity are PMC with a height of $h$. Since $h$ is much smaller than $l$ and $w$, the influence of $h$ can be neglected. Resonant energy is supplied to the resonator through the feedline. Based on the above analysis, the 

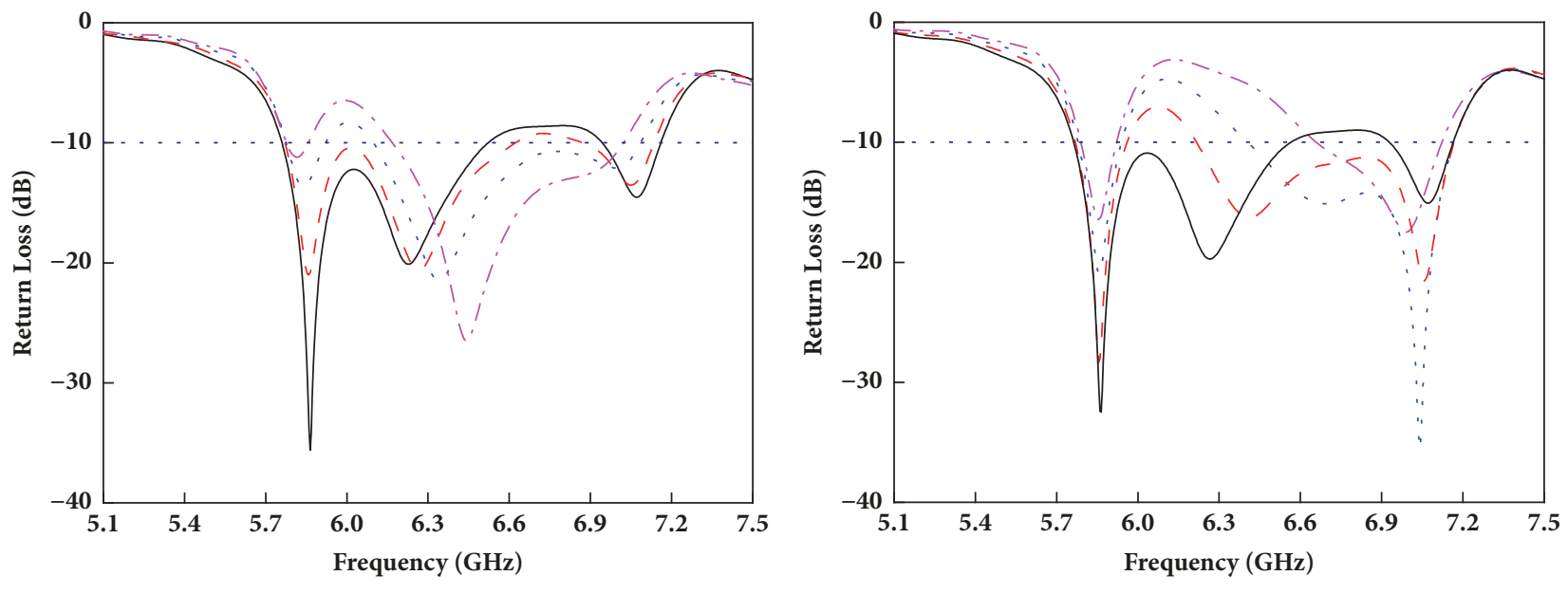

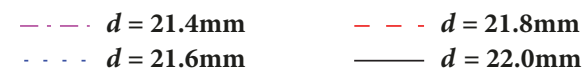

(a)

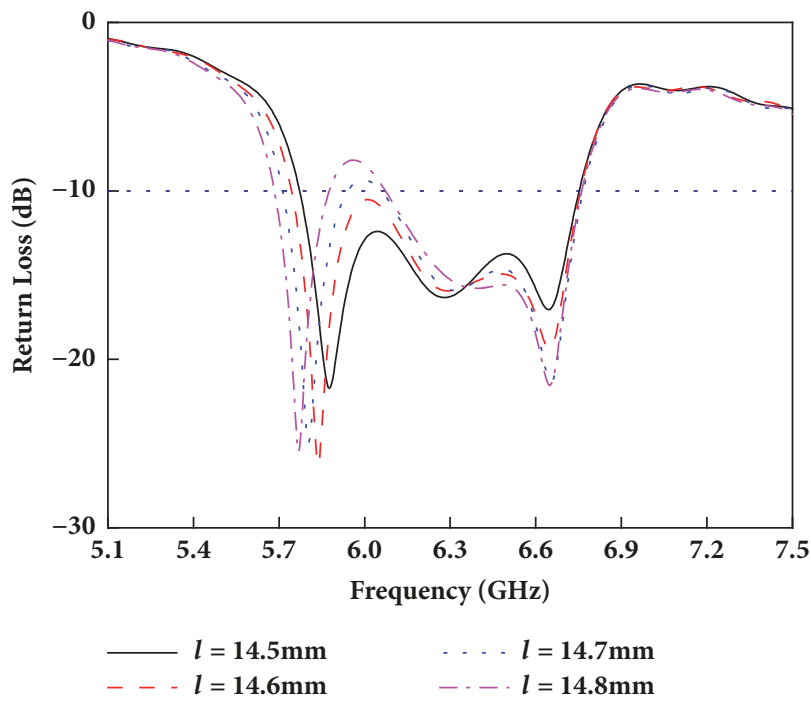

(c)

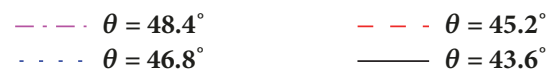

(b)

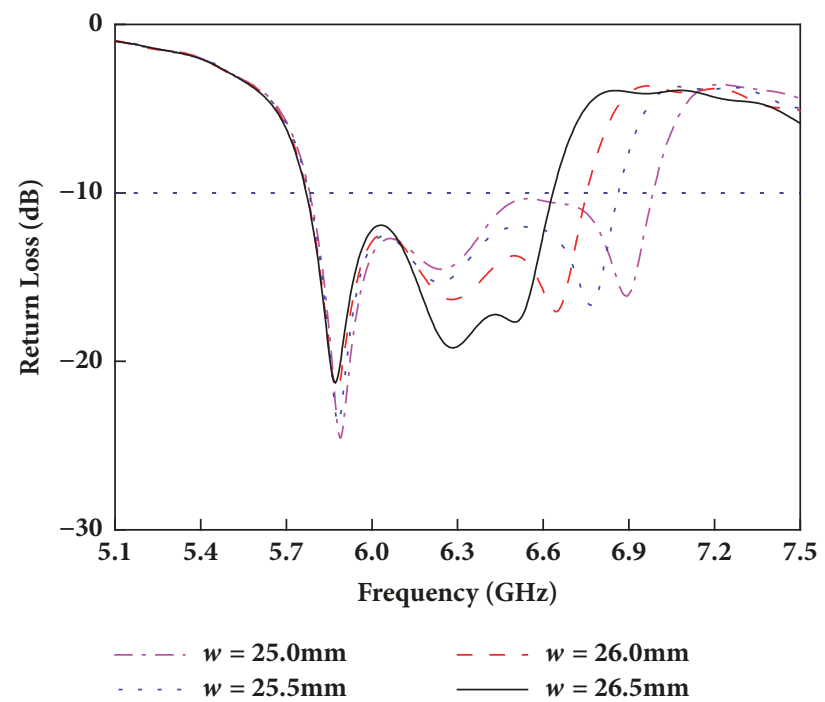

(d)

FIGURE 5: Simulated return loss of the proposed antenna with a series of (a) $d$, (b) $\theta$, (c) $l$, and (d) $w$.

inherent resonant frequency of the patch antenna operating in multiple modes is given by the following equation:

$$
f_{m n}=\frac{c}{2 \sqrt{\varepsilon_{e}}} \sqrt{\left(\frac{m}{l}\right)^{2}+\left(\frac{n}{w}\right)^{2}}
$$

where $m, n$ are nonnegative integers, $c$ is speed of light in vacuum, $\varepsilon_{e}$ is equivalent dielectric constant of the substrate, and $l$ and $w$ are the length and width of the patch, respectively. Since the thickness of the dielectric layer and the diffracted electromagnetic fields at the edges are ignored, (1) can be used for approximate predictions. The electric field between the parallel metal layers is along the $z$ axis and is cosine distributed in the $x$ and $y$ axes. While $m$ represents the number of half standing wave distributions in the $y$ direction, $n$ represents the number of half standing wave distributions in the $x$ direction. The electric field distribution is carefully observed in Figure 6. We can conclude that the antenna without CRR works at TM 10 mode and TM 02 mode corresponding to $5.88 \mathrm{GHz}$ and $6.65 \mathrm{GHz}$, respectively.

The new frequency is generated from the coupling between the radiating patch and the CRR that the resonant energy comes from the radiating patch. By carefully observing the CRR current distribution at resonance frequency, it is easy to find out that the current of the CRR acts the same as the current in the resonant cavity. The top and bottom of the CRR are cavity walls. Thus the length of cavity is $2 \times s$. According to cavity resonance theory, the condition for forming a standing wave is given by the following equation:

$$
L=\frac{|m| \lambda}{2}
$$

where $L$ is the length of the cavity, $m$ is nonzero integer. Thus the new frequency which is related to the length of the CRR can be expressed by 


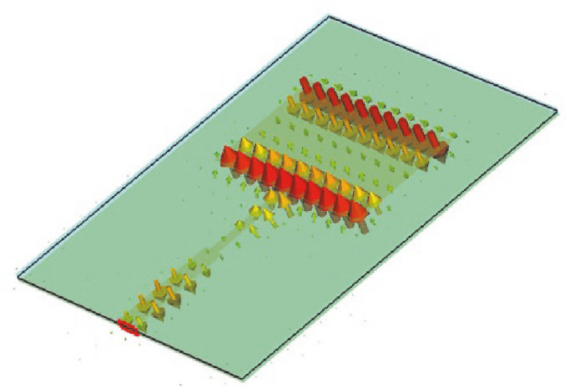

(a)

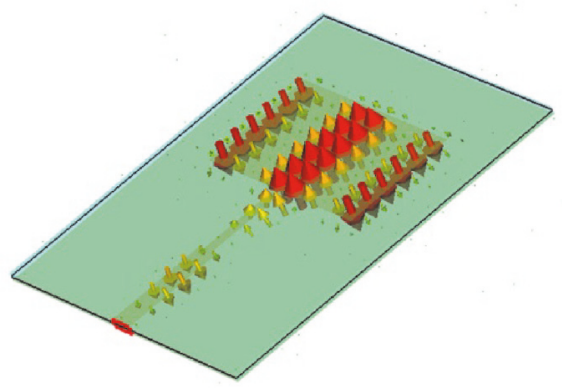

(b)

Figure 6: The electric field distribution of the antenna without CRR at (a) $5.88 \mathrm{GHz}$ and (b) $6.65 \mathrm{GHz}$.

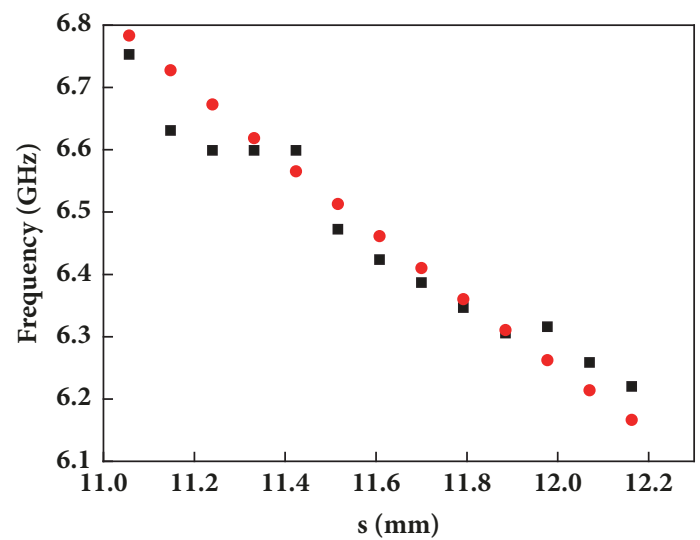

- Simulated

- Theoretical

FIGURE 7: Simulated and theoretical relationships between $s$ and the new resonant frequency.

$$
f=\frac{v}{4 n s}
$$

where $v$ is speed of light in medium, $n$ is the refractive index, and $s$ is the side length of the CRR. From (3), the resonant frequency decreases as the side length of the CRR increases. The comparison of simulated results and theoretical calculated values from (3) is depicted in Figure 7. We can see that the simulated results are in good agreement with that predicted by (3), which fully proves the feasibility of the formula.

In order to verify the simulated results, a pair of MPAs without and with the CRR were fabricated, measured, and compared. Figures 8(a) and 8(b) show the fabricated antenna without and with the CRR. As plotted in Figure 8(c), the measured bandwidth of the MPA with the CRR is 230\% larger than that of the MPA without the CRR. Figure 8(d) shows the simulated and measured return losses of the MPA with the CRR. Good agreement can be observed. It also can be found that the curve of the measured return loss shows a little shifting compared with the curve of the simulated one. Measurement imperfections and expected variations of FR4 permittivity can result in the small discrepancy.
We also investigated the radiation patterns of the MPA with CRR at their resonant frequencies, respectively. The proposed antenna radiates in the broadside direction with patterns very similar to each other in the whole band range. Figure 9 shows that the proposed antenna obtains low side-lobe and bidirectional radiation characteristics which can receive and transmit signals simultaneously. The service area is divided into small cells in a mobile communication system. In some specific cases when the mobile terminal moves along confined paths, such as tunnels, corridors, and highways, bidirectional antennas are exploited. Thus the proposed antenna may be utilized in narrow space mobile communication systems, while maintaining the small-size and low-profile antenna structure.

To achieve bandwidth enhancement, several printed slot and monopole antennas were studied. A compact monopole antenna with rectangular and triangular slots etched on the partial ground and the center of the rectangular patch achieves a wide usable fractional bandwidth of 148\% in [29]. As reported in [30], a power division network was employed in a monopole antenna, and a $10 \mathrm{~dB}$ bandwidth of $51 \%$ is obtained. An inverted L-shaped slot and an inverted Lshaped strip on the ground plane are introduced to design a wideband planar monopole antenna in [31]. However, the configurations of most of the antennas are relatively complicated. Our work presents a broadband antenna with a small size and simple structure. By comparing the antenna without CRR, the bandwidth of the proposed antenna increases by $200 \%$, which proves the feasibility and simplicity of the design.

\section{Conclusions}

A novel MPA with CRR is proposed and discussed. The CRR is etched in the ground plane, which interacts with the microstrip feedline to excite the new resonance. Parameter studies have shown that the three resonant frequencies of the proposed antenna can be adjusted separately. Therefore, the operating bandwidth of the proposed antenna can be easily regulated by changing the structural parameters of the radiating patch and the CRR. The bandwidth of the proposed antenna increases by $200 \%$ compared with that of the antenna 


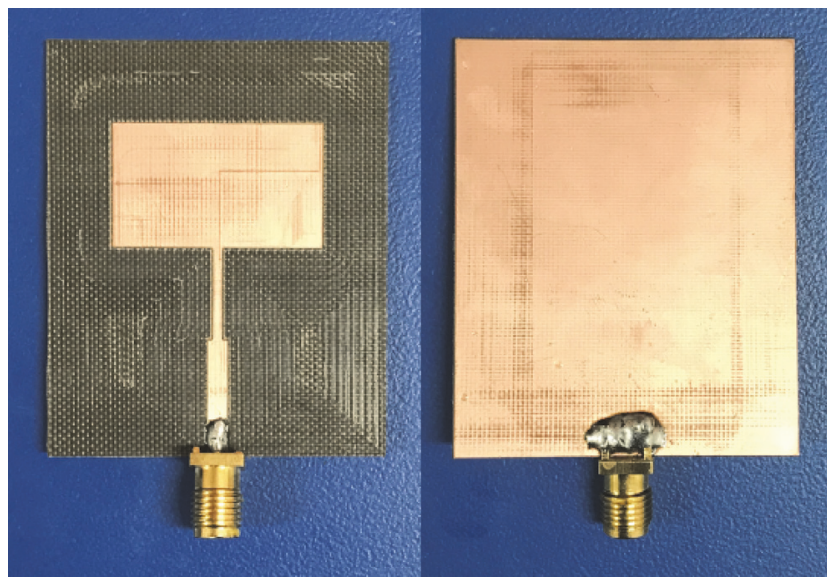

(a)

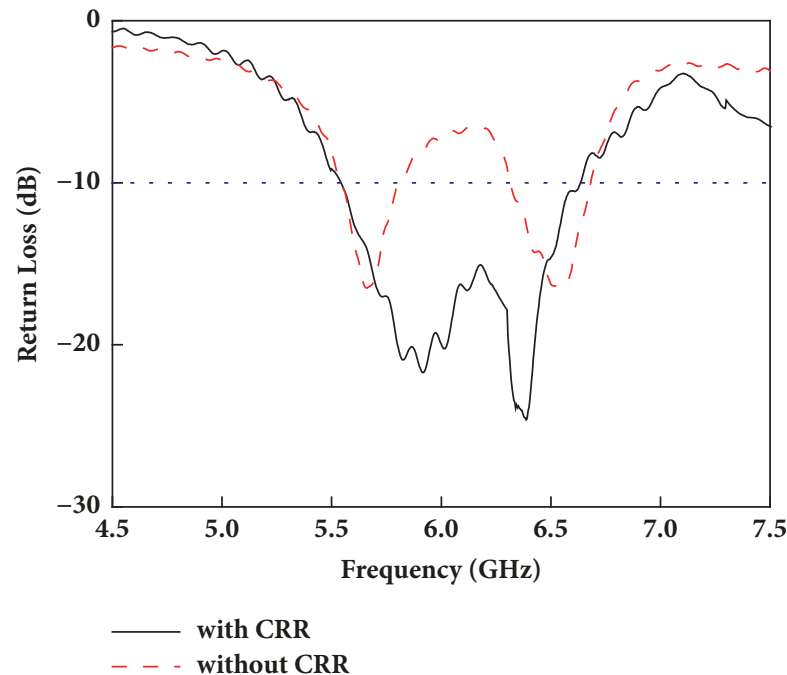

(c)

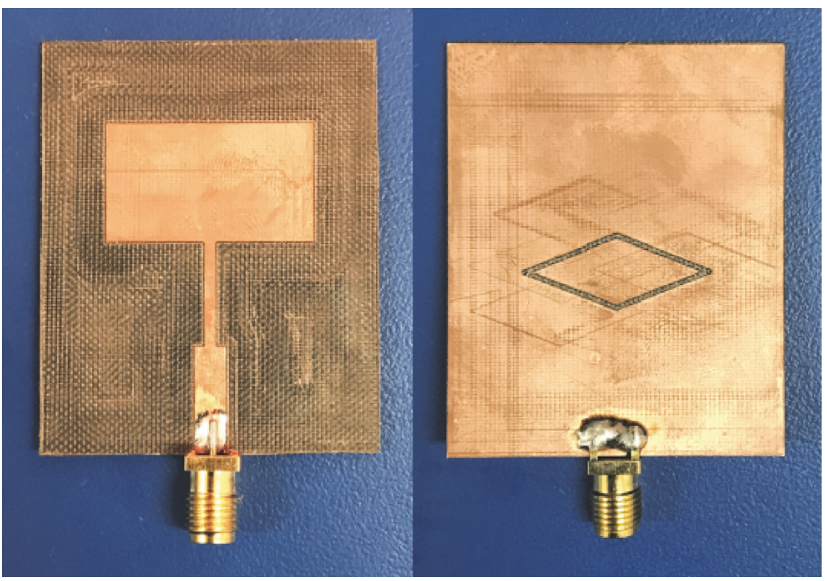

(b)

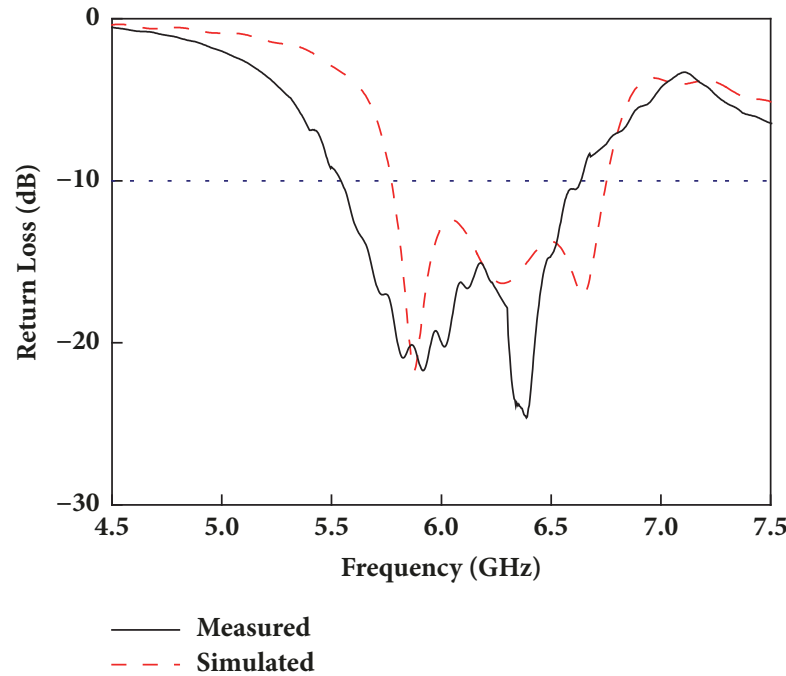

(d)

FIGURE 8: Fabricated antenna (a) without and (b) with the CRR. (c) Measured return losses of the fabricated antenna with and without the CRR. (d) Simulated and measured return losses of the MPA with the CRR.

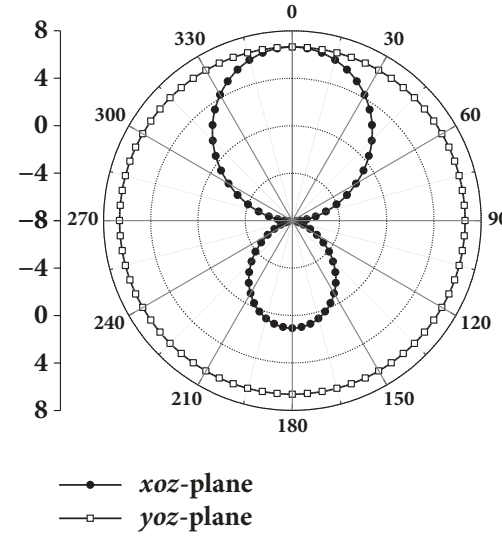

(a)

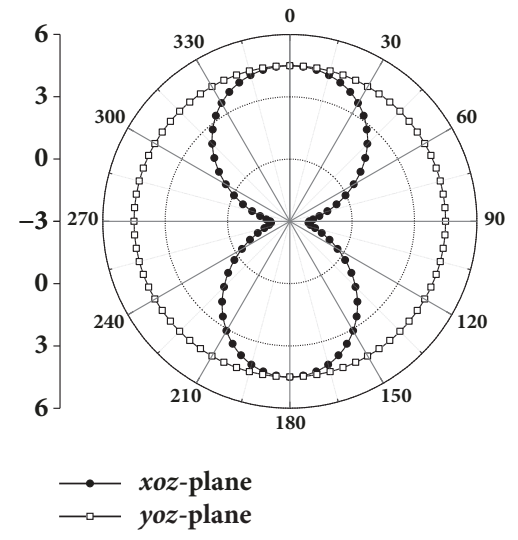

(b)

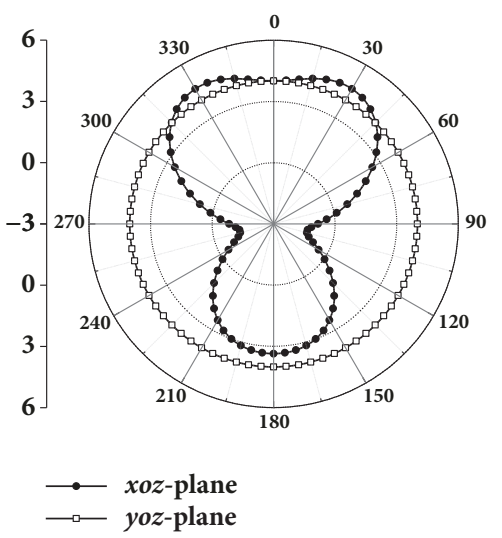

(c)

FIGURE 9: Radiation patterns of the proposed antenna at (a) 5.88, (b) 6.3, and (c) $6.6 \mathrm{GHz}$. 
without the CRR. We can conclude that the simple structure provides a way to obtain the broadband antenna.

\section{Data Availability}

The authors declare that the data supporting the findings of this study are available within the article.

\section{Conflicts of Interest}

The authors declare that there are no conflicts of interest regarding the publication of this paper.

\section{Acknowledgments}

This work was supported by the National Natural Science Foundation of China under Grant nos. 61774020, 61575028, and 61690195, Science and Technology Plan of Shenzhen City under Grant no. 201887776, and the Fund of IPOC BUPT under Grant no. IPOC2017ZT06.

\section{References}

[1] S. Haykin, "Cognitive radio: brain-empowered wireless communications," IEEE Journal on Selected Areas in Communications, vol. 23, no. 2, pp. 201-220, 2005.

[2] S. Dey and R. Mittra, "Compact microstrip patch antenna," Microwave and Optical Technology Letters, vol. 13, no. 1, pp. 1214, 1996.

[3] J. C. Xu, M. Y. Zhao, R. Zhang et al., "A wideband F-shaped microstrip antenna," IEEE Antennas and Wireless Propagation Letters, vol. 16, pp. 829-832, 2017.

[4] J. Xu, L. Tao, R. Zhang, Y. Hao, S. Huang, and K. Bi, "Broadband complementary ring-resonator based terahertz antenna," Optics Express, vol. 25, no. 15, pp. 17099-17104, 2017.

[5] Y. Hao, Q. Wang, X. Gao, S. Huang, and K. Bi, "Frequency tunable slot-coupled dielectric resonators antenna," Journal of Alloys and Compounds, vol. 702, pp. 664-668, 2017.

[6] D. Guha and Y. M. M. Antar, Microstrip and Printed Antennas: New Trends, Techniques and Applications, John Wiley and Sons, New York, NY, USA, 2011.

[7] G. Breed, "The fundamentals of patch antenna design and performance," High Frequency Electronics, pp. 48-51, 2009.

[8] M. Kara, "The resonant frequency of rectangular microstrip antenna elements with various substrate thicknesses," Microwave and Optical Technology Letters, vol. 11, no. 2, pp. 55-59, 1996.

[9] J. M. J. W. Jayasinghe and D. N. Uduwawala, "Optimization of the performance of patch antennas using genetic algorithms," Journal of the National Science Foundation of Sri Lanka, vol. 41, no. 2, pp. 113-120, 2013.

[10] M. R. Hasan and A. Al Suman, "Substrate height and dielectric constant dependent performance analysis of circular microstrip patch array antennas for broadband wireless access," American Academic and Scholarly Research Journal, vol. 4, no. 6, pp. 4349, 2012.

[11] X. H. Yi, T. Wu, Y. Wang, and M. M. Tentzeris, "Sensitivity modeling of an RFID-based strain-sensing antenna with dielectric constant change," IEEE Sensors Journal, vol. 15, no. 11, pp. 61476155, 2015.
[12] K.-L. Wong and Y.-F. Lin, "Microstrip-line-fed compact broadband circular microstrip antenna with chip-resistor loading," Microwave and Optical Technology Letters, vol. 17, no. 1, pp. 5355, 1998.

[13] A. Katyal and A. Basu, "Compact and broadband stacked microstrip patch antenna for target scanning applications," IEEE Antennas and Wireless Propagation Letters, vol. 16, pp. 381384, 2017.

[14] W. Q. Cao and W. Hong, "Bandwidth and gain enhancement for single-fed compact microstrip antenna by loading with parasitical patches," in Proceedings of the 2016 IEEE International Conference on Microwave and Millimeter Wave Technology (ICMMT), pp. 650-652, Beijing, China, June 2016.

[15] Z. Liang, J. Liu, Y. Li, and Y. Long, "A dual-frequency broadband design of coupled-fed stacked microstrip monopolar patch antenna for WLAN applications," IEEE Antennas and Wireless Propagation Letters, vol. 15, pp. 1289-1292, 2016.

[16] L. Kurra, M. P. Abegaonkar, A. Basu, and S. K. Koul, "Switchable and tunable notch in ultra-wideband filter using electromagnetic bandgap structure," IEEE Microwave and Wireless Components Letters, vol. 24, no. 12, pp. 839-841, 2014.

[17] H. A. Majid, M. K. Abd Rahim, M. R. Hamid et al., "Wideband antenna with reconfigurable band notched using ebg structure," Progress in Electromagnetics Research Letters, vol. 54, pp. 7-13, 2015.

[18] Y. J. Lee, J. Yeo, R. Mittra, and W. S. Park, "Application of electromagnetic bandgap (EBG) superstrates with controllable defects for a class of patch antennas as spatial angular filters," IEEE Transactions on Antennas and Propagation, vol. 53, no. 1, pp. 224-235, 2005.

[19] H. Attia, L. Yousefi, M. M. Bait-Suwailam, M. S. Boybay, and O. M. Ramahi, "Enhanced-gain microstrip antenna using engineered magnetic superstrates," IEEE Antennas and Wireless Propagation Letters, vol. 8, pp. 1198-1201, 2009.

[20] K. Fan, Z.-C. Hao, and Q. Yuan, "A low-profile wideband substrate-integrated waveguide cavity-backed E-shaped patch antenna for the Q-LINKPAN applications," IEEE Transactions on Antennas and Propagation, vol. 65, no. 11, pp. 5667-5676, 2017.

[21] D. V. Baelen, S. Lemey, J. Verhaevert, and H. Rogier, "A novel manufacturing process for compact, low-weight and flexible ultra-wideband cavity backed textile antennas," Materials, vol. 11, no. 1, p. 67, 2018.

[22] H. Taher, "High-performance low-pass filter using complementary square split ring resonators defected ground structure," IET Microwaves, Antennas \& Propagation, vol. 5, no. 7, pp. 771-775, 2011.

[23] J. Wang, H. Ning, and L. Mao, "A compact reconfigurable bandstop resonator using defected ground structure on coplanar waveguide," IEEE Antennas and Wireless Propagation Letters, vol. 11, pp. 457-459, 2012.

[24] R. S. Alrawashdeh, Y. Huang, M. Kod, and A. A. B. Sajak, "A broadband flexible implantable loop antenna with complementary split ring resonators," IEEE Antennas and Wireless Propagation Letters, vol. 14, pp. 1322-1325, 2015.

[25] L. Su, J. Naqui, J. Mata-Contreras, and F. Martin, "Modeling and applications of metamaterial transmission lines loaded with pairs of coupled Complementary Split-Ring Resonators (CSRRs)," IEEE Antennas and Wireless Propagation Letters, vol. 15, pp. 154-157, 2016. 
[26] A. Bage and S. Das, "A compact, Wideband waveguide bandpass filter using complementary loaded split ring resonators," Progress in Electromagnetics Research C, vol. 64, pp. 51-59, 2016.

[27] I. Yoo, M. F. Imani, T. Sleasman, and D. R. Smith, "Efficient complementary metamaterial element for waveguide-fed metasurface antennas," Optics Express, vol. 24, no. 25, pp. 2868628692, 2016.

[28] X. Cheng, D. E. Senior, C. Kim, and Y.-K. Yoon, "A compact omnidirectional self-packaged patch antenna with complementary split-ring resonator loading for wireless endoscope applications," IEEE Antennas and Wireless Propagation Letters, vol. 10, pp. 1532-1535, 2011.

[29] A. Boutejdar and W. Abd Ellatif, "A novel compact UWB monopole antenna with enhanced bandwidth using triangular defected microstrip structure and stepped cut technique," Microwave and Optical Technology Letters, vol. 58, no. 6, pp. 1514-1519, 2016.

[30] T. Kumar and A. R. Harish, "Broadband circularly polarized printed slot-monopole antenna," IEEE Antennas and Wireless Propagation Letters, vol. 12, pp. 1531-1534, 2013.

[31] S. A. Rezaeieh, A. Abbosh, and M. A. Antoniades, "Compact CPW-Fed planar monopole antenna with wide circular polarization bandwidth," IEEE Antennas and Wireless Propagation Letters, vol. 12, pp. 1295-1298, 2013. 


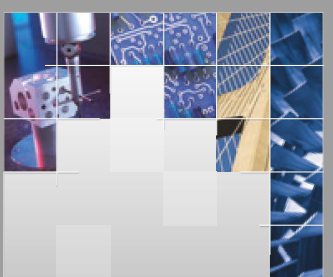

\section{Enfincering}
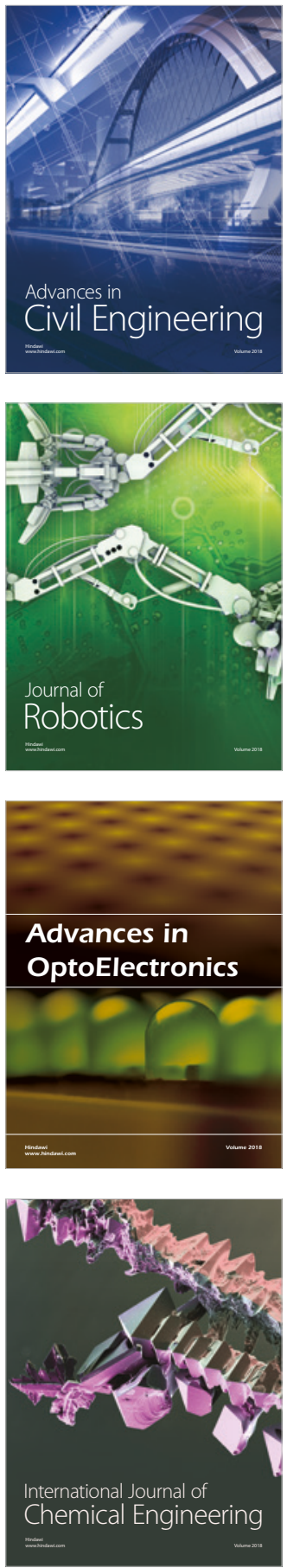

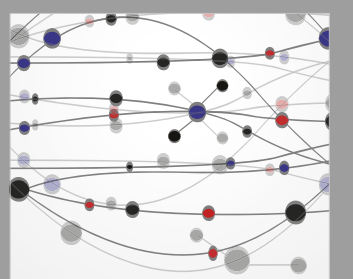

\section{Rotating \\ Machinery}

The Scientific World Journal

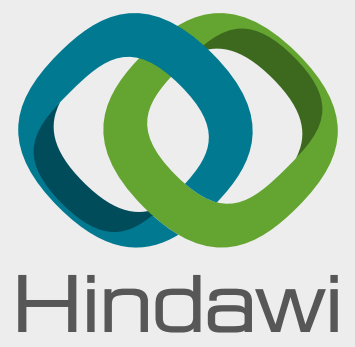

Submit your manuscripts at

www.hindawi.com
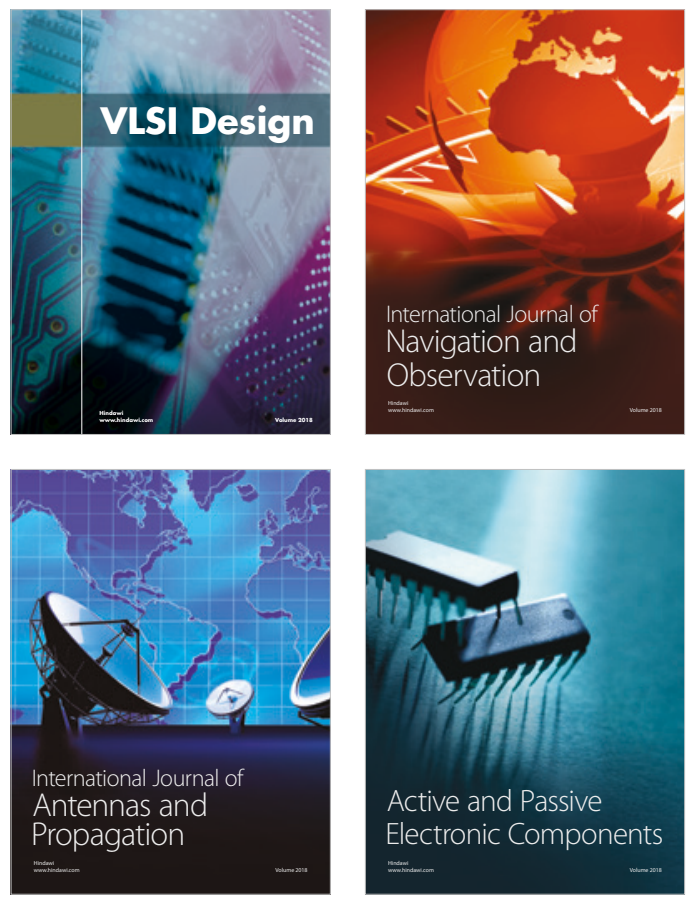
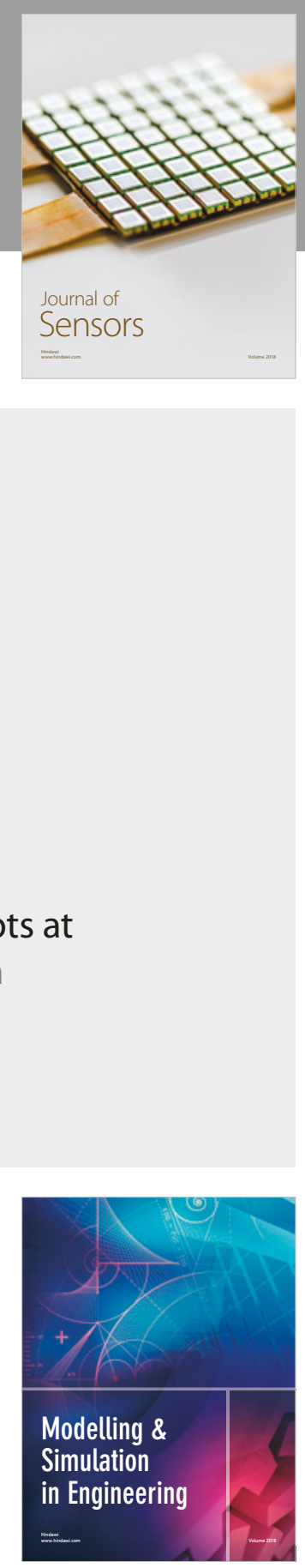

\section{Advances \\ Multimedia}
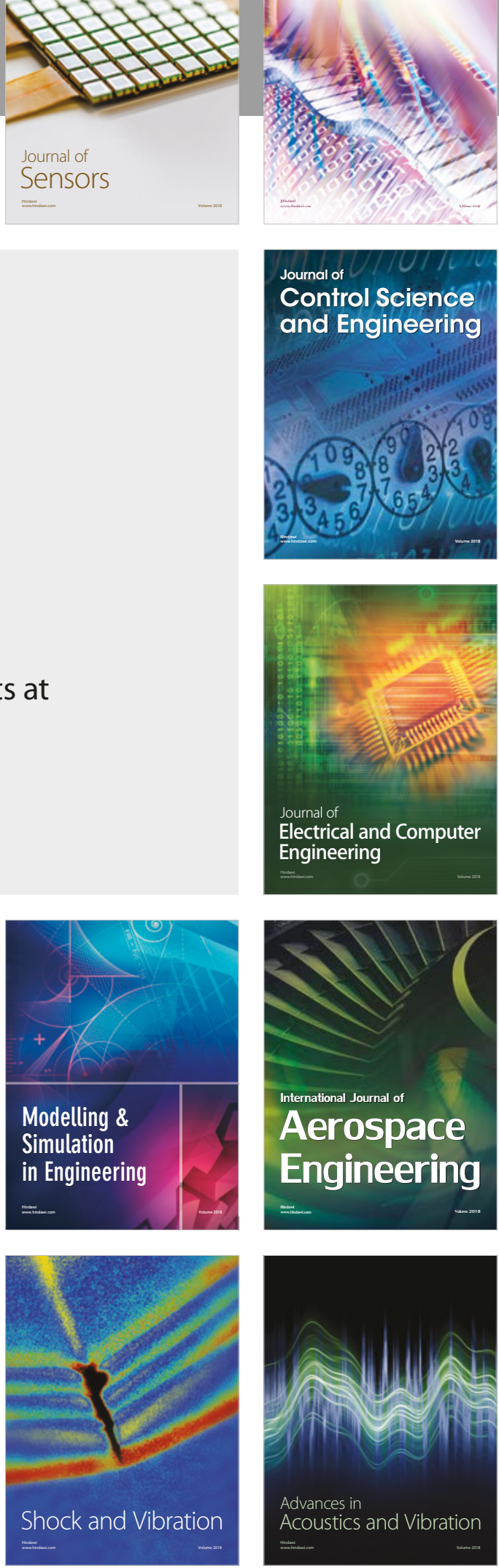